Case Report

\title{
Chondromyxoid Fibroma with Secondary Aneurysmal Bone Cyst of the Rib
}

\author{
Junichi Shimada, MD, PhD, ${ }^{1}$ Hiroaki Tsunezuka, MD, ${ }^{1}$ Kunihiko Terauchi, MD, PhD, ${ }^{1}$ \\ Masanori Shimomura, $\mathrm{MD}, \mathrm{PhD},{ }^{1}$ Eiichi Konishi, $\mathrm{MD}, \mathrm{PhD},{ }^{2}$ Motohiro Nishimura, $\mathrm{MD}, \mathrm{PhD},{ }^{3}$ \\ and Daishiro Kato, $\mathrm{MD}, \mathrm{PhD}^{1}$
}

\begin{abstract}
Chondromyxoid fibroma (CMF) is a rare, benign cartilaginous tumor, comprising less than $1 \%$ of primary bone tumors, and usually occurs in the metaphysis of a long tubular bone around the knee. We report a rare case of CMF of the rib. The patient was a 25-year-old man who visited the hospital for left upper back pain. Roentgenography revealed an 8-cm mass in the left upper lung field; computed tomography revealed a large multicystic tumor with aneurysmal bone cyst (ABC)-like features in the posterior mediastinum. ABCs are also a rare benign tumor representing $2.5 \%$ of primary bone tumors, and most of $\mathrm{ABCs}$ are located in the metaphysis of long bones and vertebrae. To the best of my knowledge, there is no report of $\mathrm{CMF}$ with secondary $\mathrm{ABC}$ of the rib. We performed total resection of the tumor. Complete tumor resection may be the best treatment option for a cure.
\end{abstract}

Keywords: chondromyxoid fibroma, rib, aneurysmal bone cyst

\section{Introduction}

Chondromyxoid fibroma (CMF) is a rare benign cartilaginous tumor, comprising less than $1 \%$ of primary bone tumors. ${ }^{1)}$ In most cases, CMFs are located in the metaphysis of long tubular bones, especially around the knee. Aneurysmal bone cyst $(\mathrm{ABC})$ also a rare benign tumor representing $2.5 \%$ of primary bone tumors, and most of $\mathrm{ABCs}$ are located in the metaphysis of long bones and

\footnotetext{
${ }^{1}$ Department of General Thoracic Surgery, Graduate School of Medical Science, Kyoto Prefectural University of Medicine, Kyoto, Kyoto, Japan

${ }^{2}$ Division of Pathology, Graduate School of Medical Science, Kyoto Prefectural University of Medicine, Kyoto, Kyoto, Japan

${ }^{3}$ Department of Thoracic Surgery, Saiseikai Suita Hospital, Suita, Osaka, Japan
}

Received: February 16, 2012; Accepted: April 5, 2012

Corresponding author: Junichi Shimada, MD, PhD. Department of General Thoracic Surgery, Graduate School of Medical Science, Kyoto Prefectural University of Medicine 465 Kajii-cho, Kawaramachi-Hirokoji, Kamigyo-ku, Kyoto, Kyoto 602-8566, Japan Email: shimajun@beige.plala.or.jp

(C)2012 The Editorial Committee of Annals of Thoracic and Cardiovascular Surgery. All rights reserved. vertebrae. ${ }^{2)}$ To the best of my knowledge, there is no report of CMF with secondary $\mathrm{ABC}$ of the rib.

\section{Case Report}

The patient was a 25 -year-old man who presented at the hospital with a 1-year history of left upper back pain. Routine roentgenography revealed an $8-\mathrm{cm}$ mass in the left upper lung field. Computed tomography (CT) revealed a plain boundary multicystic tumor, $75 \times 72 \mathrm{~mm}$ in size, on the posterior mediastinum with partition structure and calcification near the edge. Bone destruction was observed in the fourth left rib. Invasion into the surrounding tissue or the pulmonary parenchyma was not observed (Fig. 1A-1C). Low signal intensity was observed on T1-weighted magnetic resonance imaging (MRI) scans, whereas high signal intensity was observed on gadolinium-enhanced T2weighted images (Fig. 2A-2C). Positron emission tomography (PET) revealed abnormal accumulation (standardized uptake value $[\mathrm{SUVmax}]=3.4>2.5$ ) within the tumor. No tumor markers (CEA, CA19-9, AFP, NSE, and $\mathrm{HCG} \beta$ ) were elevated.

The possibility of a posterior mediastinal tumor, lung 


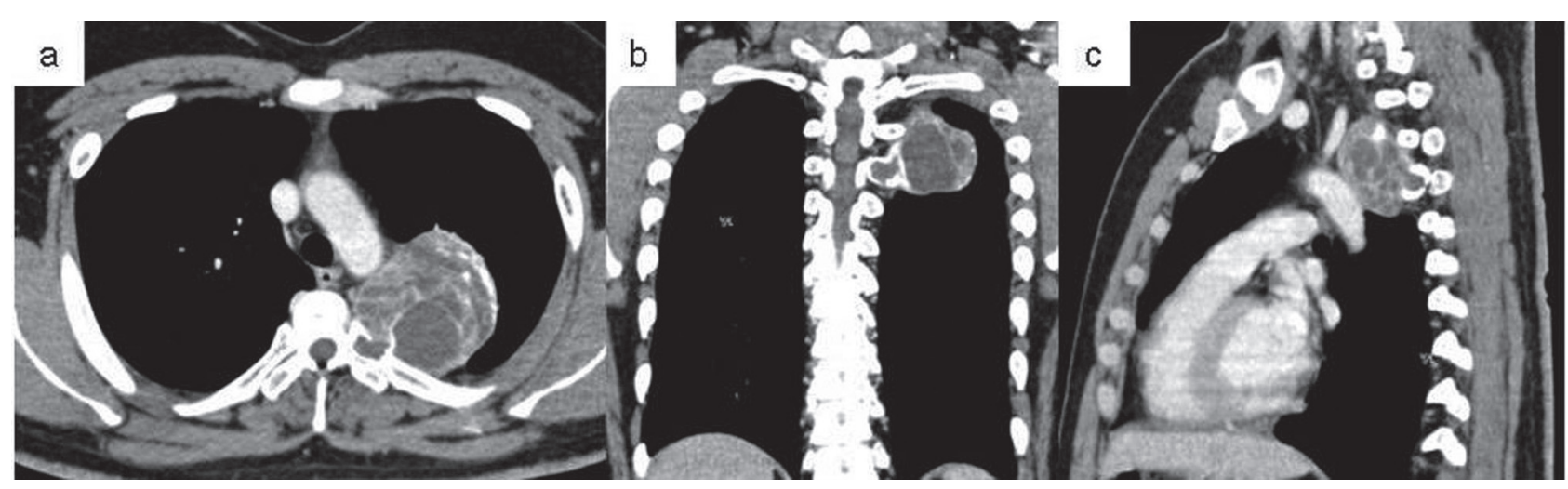

Fig. 1 Computed tomography scan shows a multicystic tumor, $75 \times 72 \mathrm{~mm}$ in size, on the posterior mediastinum with partition structure and calcification near the edge. Bone destruction was observed in the fourth left rib. Axial view (A), coronal view (B), and sagittal view $(\mathbf{C})$.

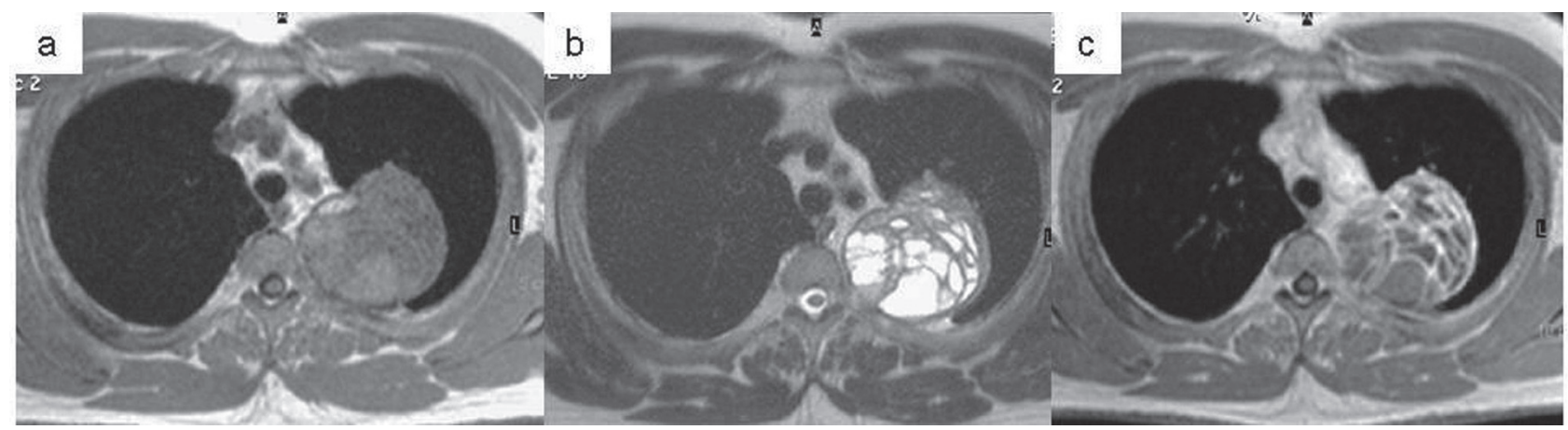

Fig. 2 Magnetic resonance imaging shows low signal intensity on T1-weighted images (A) and high signal intensity on T2-weighted images $(\mathbf{B})$; the partition structure has been enhanced by gadolinium $(\mathbf{C})$.

cancer, and the aneurysmal bone cyst (ABC)-like change was considered; therefore, we decided to remove the tumor together with the surrounding lung region and the fourth rib. The surgery was performed under general anesthesia through posterolateral thoracotomy in the right lateral position. The tumor was located in the fourth rib near the vertebrae, and it adhered to the upper lobe of the left lung. We resected the tumor together with the surrounding lung region and the fourth rib with a 2-cm margin.

Macroscopic examination revealed that the polycystic lesion was pressing into and not invading the pulmonary parenchyma of the left upper lobe (Fig. 3A). There were some hemorrhagic cystic and cavernous spaces of various sizes surrounded by fibrous septa in the tumor. The boundary between the tumor and pulmonary parenchyma was clearly demarcated by pleura. Microscopic examination revealed $\mathrm{ABC}$ projecting from bone marrow of the rib, some broken pieces of cortical bone near the edge and defect of cortex of the rib. These findings suggested $\mathrm{ABC}$ grew up and ruptured through cortex of the rib.
Stellate or spindle-shaped cells spread into the cyst wall thinly and these cells arranged in the lobules in a weakly eosinophilic chondroid-like background and these cells condensed into a hypercellular zone at the periphery of the lobules (Fig. 3B). The peripheral region was rich in small vessels and some giant cell like an osteoclast. The tumor cells were positive for $\mathrm{S100}$, and the patient was diagnosed as having chondromyxoid fibroma (CMF).

At present (4 years after surgery), the patient is doing well, with no signs of recurrence.

\section{Discussion}

CMF is a rare benign cartilaginous tumor, comprising less than $1 \%$ of all primary bone tumors. ${ }^{1)} \mathrm{CMF}$, which was first described by Jaffe and Lichtenstein in 1948, ${ }^{3)}$ has a slightly hyper incidence in men than in women and is generally detected in the second or third decade of life. In most cases, CMFs are located in the metaphysis of a long tubular bone, especially around the knee. In the remaining 

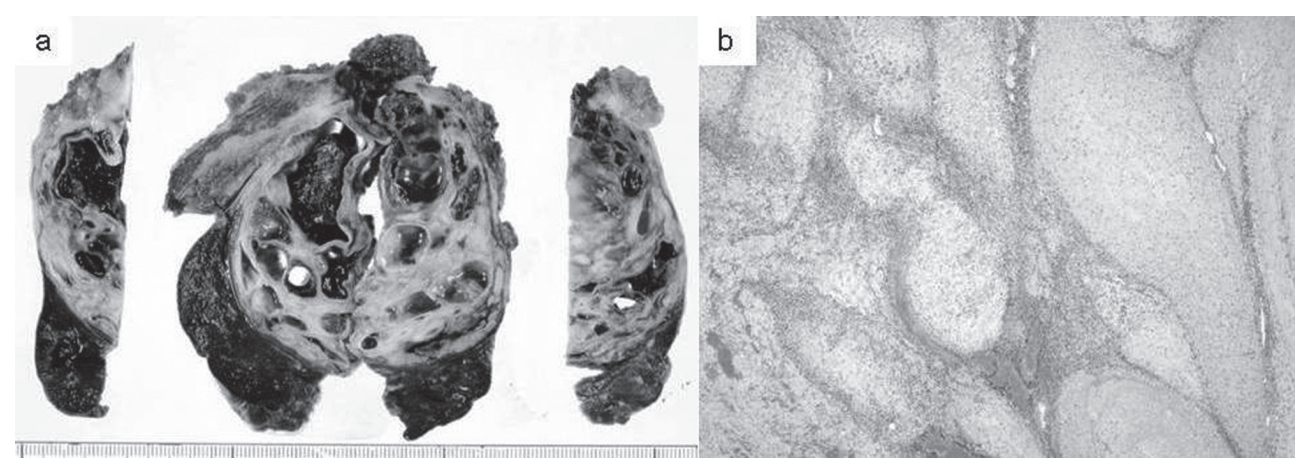

Fig. 3 Macroscopic examination shows that the polycystic lesion is clearly demarcated by pleura (A). Microscopically, stellate or spindle-shaped cells arranged in the lobules in a weakly eosinophilic chondroid-like background and these cells condensed into a hypercellular zone at the periphery of the lobules (Hematoxylin and eosin stain; $\times 20)(\mathbf{B})$.

cases, CMFs have been detected in the pelvis, ilium, or the small bones of the hands and feet. ${ }^{1,4-6)} \mathrm{CMF}$ of the rib is very rare. Recent studies suggest that aberrations in chromosome 6q13-21 are associated with local aggressiveness in cartilaginous tumors. ${ }^{5)}$ Clonal rearrangement involving $6 q 13$ especially inv (6) (p25q13) has been reported to be an additional diagnostic marker for CMFs.

In our patient, the tumor was accompanied by a large $\mathrm{ABC}$ that projected into the thoracic cavity; this made it difficult to make the final diagnosis on the basis of radiological examination. ABCs are reported to be generated by abnormal blood vessel structure due to local circulatory disturbance that in turn is caused by vessel-rich tumors in the bone. ${ }^{78}$ ABCs occur in two forms: primary and secondary. Primary ABCs arise with no known cause. Secondary ABCs are associated with highly vascularized tumors such as giant cell tumors, osteoblastomas, angiomas, chondroblastomas, traumatized bone cysts; they are less commonly associated with other diverse type of lesions such as fibrous dysplasias, fibroxanthomas, chondromyxoid fibromas, solitary bone cysts, fibrous histiocytomas and osteosarcomas. ABCs were found in $7 \%$ to $8 \%$ of CMF cases. ${ }^{1,2)}$ Although ABCs are associated with benign tumors, such as giant cell tumors, in most cases, malignant tumors, such as osteosarcomas and Ewing's sarcoma, can also cause ABCs; neoadjuvant chemotherapy should be performed in such cases. Because of cystic lesions, we were concerned regarding dissemination and expected to perform complete resection of the tumor, which had been diagnosed by frozen section at the time of surgery.

A $25 \%$ local recurrence rate after surgery has been reported in patients with CMFs, despite a benign tumor; recurrence is likely to occur in case of incomplete initial curettage. ${ }^{1)}$ Therefore, en bloc excision with enough margin is recommended to decrease the local recurrence rate of CMFs.

\section{Disclosure Statement}

There is no conflict of interest.

\section{References}

1) Wu CT, Inwards CY, O'Laughlin S, et al. Chondromyxoid fibroma of bone: a clinicopathologic review of 278 cases. Hum Pathol 1998; 29: 438-46.

2) Subach BR, Copay AG, Martin MM, et al. An unusual occurrence of chondromyxoid fibroma with secondary aneurysmal bone cyst in the cervical spine. Spine J 2010; 10: e5-9,.

3) Jaffe HL, Lichtenstein L. Chondromyxoid fibroma of bone: A distinctive benign tumor likely to be mistaken especially for chondrosarcoma. Arch Pathol (Chic). 1948; 45: 541-51.

4) Lersundi A, Mankin HJ, Mourikis A, et al. Chodromyxoid fibroma:A rarely encountered and puzzling tumor. Clin Orthop Rela Res 2005; 439: 171-5.

5) Armah HB, McGough RL, Goodman MA, et al. Chondromyxoid fibroma of rib with a novel chromosomal translocation: a report of four additional cases at unusual sites. Diagn Pathol 2007; 2: 44.

6) Baker AC, Rezeanu L, O'Laughlin S, et al. Juxtacortical chondromyxoid fibroma of bone: a unique variant: a case study of 20 patients. Am J Surg Pathol 2007; 31: 1662-8.

7) Kransdorf MJ, Sweet DE. Aneurysmal bone cyst: concept, controversy, clinical presentation, and imaging. Am J Roentgenol 1995; 164: 573-80.

8) Mankin HJ, Hornicek FJ, Ortiz-Cruz E, et al. Aneurysmal bone cyst: a review of 150 patients. J Clin Oncol 2005; 23: 6756-62. 